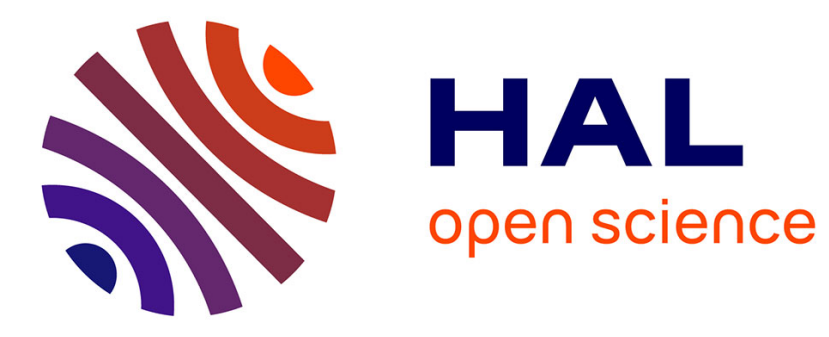

\title{
Impuretés solubles contenues dans la glace de l'Antarctique
}

\author{
C. Lorius, G. Baudin, J. Cittanova, R. Platzer
}

\section{To cite this version:}

C. Lorius, G. Baudin, J. Cittanova, R. Platzer. Impuretés solubles contenues dans la glace de l'Antarctique. Tellus A, 1969, 21 (1), pp.136 - 148. 10.3402/tellusa.v21i1.10064 . hal-03402792

\section{HAL Id: hal-03402792 \\ https://hal.science/hal-03402792}

Submitted on 25 Oct 2021

HAL is a multi-disciplinary open access archive for the deposit and dissemination of scientific research documents, whether they are published or not. The documents may come from teaching and research institutions in France or abroad, or from public or private research centers.
L'archive ouverte pluridisciplinaire HAL, est destinée au dépôt et à la diffusion de documents scientifiques de niveau recherche, publiés ou non, émanant des établissements d'enseignement et de recherche français ou étrangers, des laboratoires publics ou privés. 


\section{Tellus}

\section{Impuretés solubles contenues dans la glace de l'Antarctique}

\section{Lorius, G. Baudin, J. Cittanova \& R. Platzer}

To cite this article: C. Lorius, G. Baudin, J. Cittanova \& R. Platzer (1969) Impuretés solubles contenues dans la glace de l'Antarctique, Tellus, 21:1, 136-148, DOI: 10.3402/tellusa.v21i1.10064

To link to this article: https://doi.org/10.3402/tellusa.v21i1.10064
(c) 1969 The Author(s). Published by Taylor and Francis Group LLC

曲 Published online: 15 Dec 2016.

Submit your article to this journal $\widetilde{ }$

Џ Article views: 34

Q View related articles $\sqsubset$ 


\title{
Impuretés solubles contenues dans la glace de l'Antarctique
}

\author{
Par C. LORIUS Centre d'Etudes Glaciologiques des Régions Arctiques et Antarctiques, et G. BAUDIN, \\ J. CITTANOVA et R. PLATZER, Service d'Etudes Analytiques, Saclay
}

(Manuscrit reçu le 16 juin 1967)

\begin{abstract}
Ionic concentrations in the ice (down to 100 meters deep) and in the firn of the costal region of Terre Adelie have been studied. The results show that the concentration found in the upper layers of the ice is sensibly the same as that found in locally accumulated layers of the firn. The principal ions, of which average concentration is always less than $1 \mathrm{mg} / \mathrm{l}$, are in order of their importance $\mathrm{Cl}, \mathrm{Na}$, and $\mathrm{SO}_{4}$ and then $\mathrm{K}$ and $\mathrm{Ca}$. The ice in lower layers, originating by way of ice flow from inland area of Anterctica, contains three times less impurities. The concentration of $\mathrm{SO}_{4}$ ions then becomes predominant $(0.24 \mathrm{mg} / \mathrm{l})$. The possible origin of these different ions is discussed. Important variations in their concentration observed at one station do not seem to be linked to a seasonal phenomenom.

On the base of published data, it has been estimated that every year approximately $4 \cdot 10^{6}$ tons of soluble impurities are deposited on Antarctica. These impurities take part in a cycle, but the ice of this continent could contain up to $19 \cdot 10^{9}$ tons of soluble salts.
\end{abstract}

\section{Introduction}

Les couches de neige qui s'accumulent dans les régions froides renferment un certain nombre de substances, le plus souvent présentes à l'état de traces. Dans les régions polaires où la fusion est pratiquement inexistante, ces substances sont conservées lors de la transformation du névé en glace, puis lors de l'écoulement de cette glace. De plus, et en particulier dans l'Antarctique, les risques de pollution par apport de matériaux dus à l'activité de l'homme sont très réduits par suite de l'éloignement des centres de cette activité. Pour ces différentes raisons, ces régions se prêtent particulièrement bien à l'étude des impuretés naturelles contenues dans la glace, d'une part en fonction de la situation géographique et, d'autre part, en fonction du temps. Pour ce dernier point, les archives constituées par exomple dans l'Antarctique, depuis des dizaines de milliers d'années, sont une source d'information pratiquement encore inexplorée.

Les études que nous avons entreprises portent, d'une façon générale, sur la détermination de la composition chimique (éléments gazeux et solides, solubles ou insolubles) et des teneurs isotopiques (isotopes stables et radioactifs); le présent travail concerne la concentration des principaux ions dans l'eau de fusion provenant d'échantillons de nóvé et de glace prélevés dans l'Antarctique. Cette concentration est liée à un ensemble de phénomènes très complexes que nous ne ferons que rappeler ici. Tout d'abord, ces ions peuvent provenir de substances ayant une origine différente, extra-terrestre ou terrestre. Dans ce dernier cas, ces substances peuvent être naturelles ou artificielles. De plus, elles peuvent se déposer par des mécanismes très différents (Junge, 1963) tels que par précipitation, sédimentation ou impact, absorption ou réaction chimique. Enfin, leur concentration dépend non seulement de leur origine ou de leur mode de déposition mais aussi de l'origine et de l'évolution des masses d'air auxquelles elles sont liées.

Pourtant l'intérêt des informations que l'on peut espérer recueillir justifie que de telles études soient entreprises; ces informations touchent en effet à différents domaines tels que :

- la glaciologie : effet géographique permettant de caractériser l'origine de la glace, variation saisonnière permettant de différencier les couches de neige et donc de connaître l'accumulation;

- la météorologie : circulation et échange des masses d'air à partir de traceurs chimiques, formation des précipitations; 


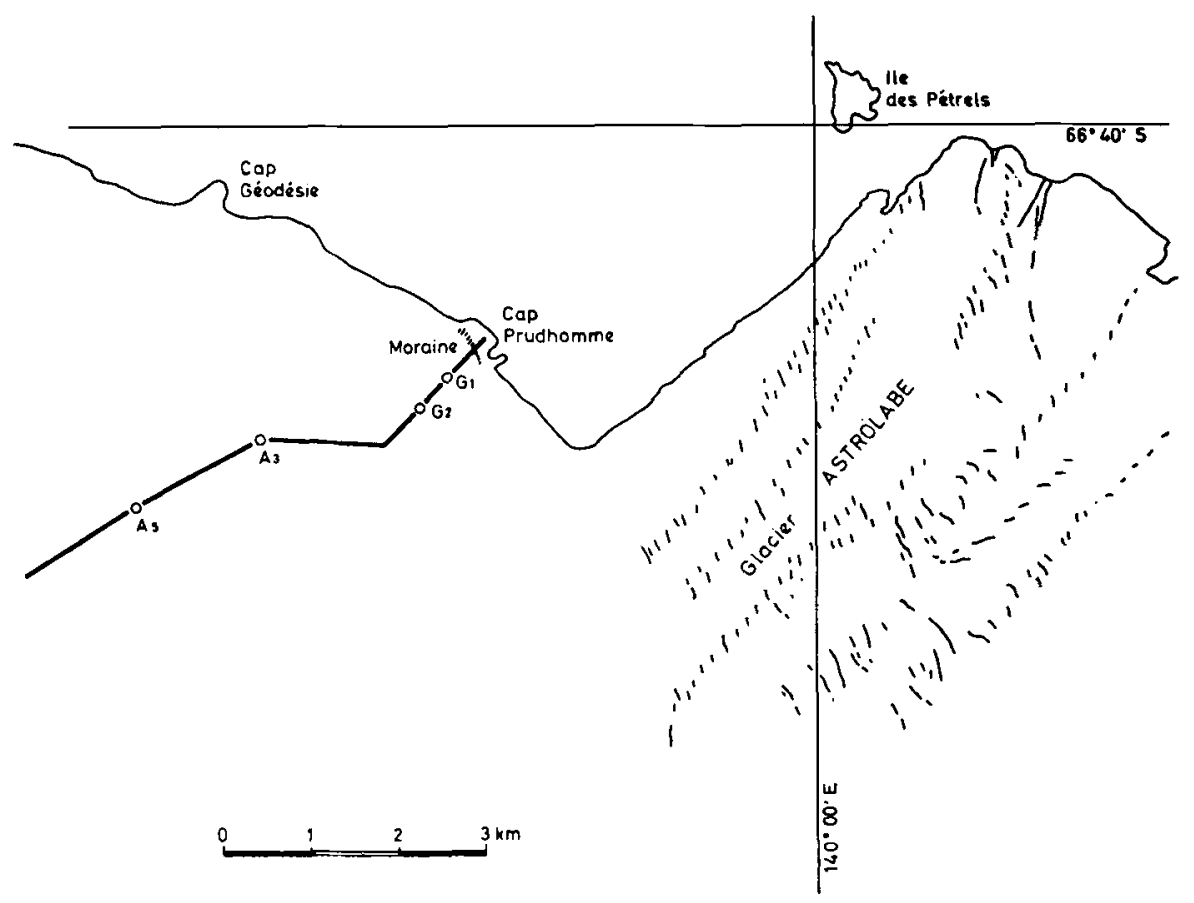

Fig. 1. Stations de prélèvements en Terre Adélie.

- la géochimie : cycle et budget de différentes substances, variations dans le temps;

- l'étude de la haute atmosphère : matériaux d'origine extraterrestre, isotopes produits par le rayonnement cosmique.

\section{Prélèvements - méthodes de mesure}

\subsection{Campagnes de prélèvements}

Des prélèvements ont été effectués dans la région côtière de Terre Adélie, au voisinage de la base Dumont d'Urville $\left(66^{\circ} 40^{\prime} \mathrm{S}-140^{\circ} 01^{\prime} \mathrm{E}\right)$ au cours de différentes campagnes organisées par les Expéditions Polaires Françaises dans le cadre des Terres Australes et Antarctiques Françaises (Fig. 1).

La première série d'échantillons provient d'un carottage (G 1 ) de $97,8 \mathrm{~m}$ de profondeur réalisé dans la glace, à proximité de la moraine située vers Cap Prudhomme. A cette station, l'accumulation et l'ablation sont pratiquement nulles et l'on a rencontré, lors du carottage, de la glace blanche à l'exception d'une couche de 11 m d'épaisseur située entre 83,3 et $94,3 \mathrm{~m}$ de profondeur qui contient des poussières morainiques et d'une couche d'environ $2 \mathrm{~m}$ de glace bleue située au contact du socle rocheux. La température croît de la surface $\left(-11^{\circ} \mathrm{C}\right)$ vers le fond $\mathrm{du}$ forage $\left(-8^{\circ} \mathrm{C}\right)$.

Les autres séries proviennent de carottages effectués dans le névé aux stations A 3 et A5. Ces deux stations sont situées à 2,8 et $4,5 \mathrm{~km}$ de Cap Prudhomme, à des altitudes de 220 et $230 \mathrm{~m}$. La température moyenne annuelle mesurée est de l'ordre de $-15^{\circ} \mathrm{C}$ et l'accumulation moyenne au cours des dernières années est d'environ $45 \mathrm{~cm}$ de névé par an.

Les carottes recueillies ont un diamètre de $60 \mathrm{~mm}$ (G 1) et de $76 \mathrm{~mm}$ (A3 ot A5); après un premier nettoyage pour lequel on utilisait notamment des gants de caoutchouc, elles ont été emballées dans des enveloppes en polyéthylène soudées puis placées à l'intérieur de tubes cartonnés. Les opérations effectuées sur le terrain sont ainsi réduites au minimum. De plus, pour éviter toute évolution de composition, la température de ces échantillons est demeurée toujours nettement en dessous de $0^{\circ} \mathrm{C}$ tant au cours du forage que des opérations de transport; aucun réfrigérant susceptible de les polluer n'a été utilisé. 
Tableau 1. Sensibilité et précision des mesures

\begin{tabular}{lccccccc}
\hline & $\mathrm{Cl}$ & $\mathrm{Na}$ & $\mathrm{Mg}$ & $\mathrm{K}$ & $\mathrm{Ca}$ & $\mathrm{SO}_{4}$ & $\mathrm{SiO}_{2}$ \\
\hline Sensibilité (mg/l) & 0,2 & $\mathbf{0 , 0 0 5}$ & $\mathbf{0 , 0 0 5}$ & $\mathbf{0 , 0 1}$ & $\mathbf{0 , 0 1}$ & $\mathbf{1 0}$ & $\mathbf{0 , 2}$ \\
Précision (\%) & $\mathbf{3 - 1 0}$ & $\mathbf{3}$ & $\mathbf{5}$ & $\mathbf{3}$ & $\mathbf{3}$ & $\mathbf{1 5}$ & $\mathbf{2 - 5}$ \\
\hline
\end{tabular}

\subsection{Préparation des échantillons}

Les échantillons destinés à l'analyse chimique ont été préparés en chambre froide; la partie extérieure a été éliminée par un grattage énergique, les prélèvements propres étant ensuite placés dans des récipients étanches en polyéthy. lène. Les outils, récipients et gants utilisés pour les manipulations étaient soigneusement nettoyés à l'eau bi-distillée avant shaque opération. Les échantillons étaient ensuite transportés immédiatement dans les laboratoires de chimie. Pour le dosage de la silice en particulier, ils ont été conservés sous forme solide jusqu'au moment de la mesure.
Les prélèvements choisis se répartissent de la façon suivante :

- ̀̀ la station A 3, 16 échantillons contigus prélevés vers la surface sur une profondeur d'environ $1.75 \mathrm{~m}$;

- à la station A 5, 4 échantillons moyens représentant chacun environ $1 \mathrm{~m}$, répartis entre la surface et $10 \mathrm{~m}$ de profondeur;

- au point de carottage G1, 6 échantillons moyens représentant chacun environ $1 \mathrm{~m}$; de plus, en six autres profondeurs, on a effectué une étude fine des variations sur des prélèvements contigus ayant entre 3 et $10 \mathrm{~cm}$ d'épaisseur, le tout représentant 78 échantillons.

Tableau 2. Concentrations dans

\begin{tabular}{|c|c|c|c|c|c|c|c|c|}
\hline Prof. (m) & $\mathbf{N}$ & $\mathrm{Cl}$ & $\mathrm{Na}$ & $\mathbf{K}$ & $\mathrm{Ca}$ & $\mathbf{M g}$ & $\mathrm{SO}_{4}$ & $\mathrm{SiO}_{2}$ \\
\hline \multicolumn{9}{|l|}{$G 1$} \\
\hline $13,2-14$ & 1 & 0,99 & 0,66 & 0,10 & 0,16 & 0,07 & 0,48 & 0,02 \\
\hline $19,5-20,5$ & 20 & 1,18 & 0,80 & 0,15 & 0,36 & 0,08 & & \\
\hline $20,5-21,6$ & 9 & 0,70 & 0,41 & & & 0,05 & & \\
\hline $21,6-22,8$ & 22 & 0,61 & 0,42 & & & 0,06 & & \\
\hline $24,6-25,4$ & 1 & & & & & & & 0,08 \\
\hline $25,4-26,3$ & 1 & & & & & & & 0,09 \\
\hline $36,8-37,5$ & 1 & 0,46 & 0,65 & 0,45 & 0,11 & 0,03 & 0,42 & 0,02 \\
\hline $41,8-43,8$ & 1 & $<0,17$ & 0,12 & 0,09 & 0,03 & 0,01 & 0,28 & 0,02 \\
\hline $47,2-50$ & 1 & $<0,17$ & 0,07 & 0,09 & 0,05 & 0,01 & 0,22 & 0,04 \\
\hline $53,7-54,4$ & 1 & & & & & & & 0,02 \\
\hline $55,4-55,3$ & 1 & & & & & & & 0,11 \\
\hline $55,8-57,2$ & 9 & $0,19-0,29$ & 0,21 & $0,10-0,12$ & $0,02-0,10$ & $0,005-0,01$ & & \\
\hline $57,2-57,8$ & 13 & $<0,17$ & 0,04 & $<0,10$ & $<0,10$ & 0,01 & & \\
\hline $67,9-68,7$ & 1 & $<0,17$ & 0,12 & 0,13 & 0,06 & 0,01 & 0,25 & 0,04 \\
\hline $74,9-75,4$ & 1 & $<0,17$ & 0,13 & 0,17 & 0,05 & 0,01 & 0,20 & 0,06 \\
\hline $92,2-92,9$ & 6 & $0,16-0,25$ & 0,35 & 0,42 & 0,49 & 0,22 & & \\
\hline \multicolumn{9}{|l|}{$A 3$} \\
\hline $0-1,75$ & 16 & $0,79(10)$ & 0,94 & $0,24-0,26$ & & 0,11 & & \\
\hline \multicolumn{9}{|l|}{$A 5$} \\
\hline Entre 0 et 10 & 4 & $0,47-0,51$ & 0,46 & 0,15 & 0,09 & 0,05 & 0,33 & 0,03 \\
\hline \multicolumn{2}{|l|}{ Esu de $\operatorname{mer}^{a} \mathrm{~g} / \mathrm{l}$} & 18,98 & 10,56 & 0,38 & 0,40 & 1,27 & 2,65 & \\
\hline
\end{tabular}

${ }^{a}$ Junge, 1963. 


\subsection{Méthodes de mesure}

La détermination de la concentration des différents éléments a été effectuée sur l'eau non acidifiée, immédiatement après fusion de la glace placée dans des récipients en polyéthylène étanches et préalablement soigneusement nettoyés; on a vérifié qu'une eau bi-distillée ayant séjourné 8 jours dans le récipient ne présente aucune contamination, en particulier pour le chlore.

Les analyses des éléments $\mathrm{Na}, \mathrm{Mg}, \mathrm{K}, \mathrm{Ca}, \mathrm{Cl}$, sont effectuées sans concentration sur $20 \mathrm{ml}$ de solution; les sulfates sont dosés sur une solution concentrée 40 fois (1 litre réduit à $25 \mathrm{ml}$ ) et les autres impuretés sur une eau concentrée 10 fois. Pour la silice, le prélèvement sous forme de glace $(50 \mathrm{~g})$ est fondu directement dans le récipient d'analyse en vue d'éviter les pertes par adhérence sur les parois.

Les méthodes utilisées sont les suivantes :

- Spectrophotométrie d'absorption atomique pour les éléments $\mathrm{Na}, \mathrm{Mg}, \mathrm{K}$ et $\mathrm{Ca}$.

- Electrochimie (chronoampérométrie linéaire) pour le chlore; les carbonates, qu'il est im. possible de différencier des chlorures par cette méthode, sont éliminés par barbotage d'argon dans la solution préalablement acidifiée par l'acide perchlorique.

- Turbidimétrie du sulfate de baryum pour les sulfates (Charlot, 1966).

- Spectrophotométrie d'absorption du complexe silicomolybdique réduit par le sulfate ferreux en milieu oxalique (Charlot, 1966) pour la silice.

- Spectrographie d'émission optique ("copper spark " ou "graphite spark ") pour les impuretés métalliques. L'enregistrement des spectres effectué sur plaque photographique met en évidence tous les éléments métalliques au niveau de quelques parties par million (méthode $\mathrm{Ce}$ $\operatorname{tama} n^{\circ} 76$ ).

- Oxydation par le permanganate de potassium pour les matières organiques.

L'absence de résultats concernant les carbonates est due à la difficulté d'interprétation, notamment en ce qui concerne la différenciation des ions provenant des solides et ceux provenant des gaz dissous. D'une manière générale, on a opéré dans des conditions telles que seuls les

la glace en Terre Adélie ( $m g / l)$

\begin{tabular}{|c|c|c|c|c|c|c|}
\hline $\mathrm{Cl} / \mathrm{Na}$ & $\mathrm{Cl} / \mathrm{Ca}$ & $\mathrm{Cl} / \mathbf{M g}$ & $\mathrm{Cl} / \mathrm{K}$ & $\mathrm{K} / \mathrm{Na}$ & $\mathbf{M g} / \mathbf{N a}$ & $\mathrm{SO}_{4} / \mathrm{Na}$ \\
\hline $\begin{array}{l}1,51(1) \\
1,56(20) \\
1,71(9) \\
1,63(22)\end{array}$ & $\begin{array}{l}6,19(1) \\
3,70(20)\end{array}$ & $\begin{array}{l}13,38(1) \\
15,75(20) \\
15,15(9) \\
10,65(22)\end{array}$ & $\begin{array}{l}9,90(1) \\
8,57(20)\end{array}$ & $\begin{array}{l}0,16(1) \\
0,20(20) \\
0,17(22)\end{array}$ & $\begin{array}{ll}0,11 & (1) \\
0,11 & (20) \\
0,12 & (9)\end{array}$ & $0,73(1)$ \\
\hline 0,71 (1) & $4,18(1)$ & $15,33(1)$ & $1,02(1)$ & $\begin{array}{l}0,68(1) \\
0,73(1) \\
1,31(1)\end{array}$ & $\begin{array}{l}0,04(1) \\
0,11(1) \\
0,17(1)\end{array}$ & $\begin{array}{l}0,65(1) \\
2,33(1) \\
3,14(1)\end{array}$ \\
\hline $1.32(4)$ & $3,91(2)$ & $41,8(4)$ & $2,91(3)$ & $\begin{array}{l}0,46(6) \\
1,10(1) \\
1,29(1)\end{array}$ & $\begin{array}{l}0,04(4) \\
0,09(1) \\
0,11(1)\end{array}$ & $\begin{array}{l}2,08(1) \\
1,54(1)\end{array}$ \\
\hline $0,72(10)$ & & $6,01(10)$ & $3,59(8)$ & $0,33(8)$ & $0,12(16)$ & \\
\hline $1,12(3)$ & $6,57(3)$ & $10,56(3)$ & $3,88(3)$ & $0,35(4)$ & $0,11(4)$ & $0,82(4)$ \\
\hline 1,80 & 47,45 & 14,95 & 49,95 & 0,04 & 0,12 & 0,25 \\
\hline
\end{tabular}

Tellus XXI (1969), 1 
éléments en solution sont dosés, à l'exception toutefois de la silice dont la teneur correspond à la somme des fractions solubles et insolubles.

La sensibilité (sans tenir compte de la concentration effectuée sur la solution de départ) et la précision (dans le domaine des échantillons analysés) sont les suivantes : (Tableau 1).

\section{Résultats et interprétation}

La concentration des ions varie dans d'assez larges mesures entre les différentes stations et, pour une même station, en fonction de la profondeur, même dans le cas d'échantillons contigus. Dans le but d'étudier les phénomènes d'une façon représentative, nous nous sommes attachés, tout d'abord, à la détermination des valeurs moyennes.

\subsection{Valeurs moyennes}

Les valeurs données Tableau 2 ont été soit obtenues directement à partir d'échantillons représentant environ $1 \mathrm{~m}$ de profondeur, soit calculées à partir des mesures individuelles dans le cas d'échantillons plus fins; les rapports de concentration sont alors la moyenne des rapports calculés pour chacune des mesures où l'on a des valeurs exactement déterminées. L'examen de ce tableau conduit aux constatations suivantes :

- Dans le cas des stations A 3 et A5, la concentration des différents éléments est inférieure à $1 \mathrm{mg} / \mathrm{l}$, les ions les plus importants étant $\mathrm{Cl}$, $\mathrm{Na}$ et $\mathrm{SO}_{4}$, puis $\mathrm{K}, \mathrm{Ca}$ et $\mathrm{Mg}$.

- Dans le cas du forage $\mathrm{G} 1$, la concentration des différents éléments décroît en fonction de la profondeur. Cette décroissance, particulièrement marquée pour les ions $\mathrm{Cl}$ et $\mathrm{Na}$, est caractérisée par une variation très nette aux environs de $40 \mathrm{~m}$. Les concentrations demeurent ensuite très faibles sauf pour le dernier échantillon (92 $\mathrm{m})$ situé dans la partie du forage renfermant des poussières morainiques. L'accroissement des teneurs est alors dî au passage en solution d'une partie de ces poussières. Dans les premiers $40 \mathrm{~m}$, les teneurs trouvées sont du même ordre que celles des stations $\mathbf{A} 3$ et $\mathbf{A} 5$, tandis qu'entre 40 et $80 \mathrm{~m}$, les ions $\mathrm{SO}_{4}$ deviennent prédominants (Fig. 2).

- Les teneurs en silice sont très faibles pour les différentes stations.
Ces différentes remarques sont confirmées par le calcul des valeurs moyennes indiquées dans le Tableau 3. L'écart observé entre les concentrations mesurées pour la partie inférieure de Gl d'une part et pour les stations A 3, A 5 et partie supérieure de G 1 d'autre part peuvent cependant s'expliquer si l'on rappelle que pour G1, la mesure de la concentration en deutérium a conduit (Merlivat, Lorius \& Nief, 1966; Merlivat, Lorius, Majzoub, Nief \& Roth, 1966) aux résultats suivants : entre 0 et $32 \mathrm{~m}$, la glace est d'origine locale, c'est-à-dire qu'elle provient de la transformation de précipitations déposées jusqu'à une distance de $100 \mathrm{~km}$ environ. Une zone de transition existe ensuite sur une profondeur de l'ordre de $20 \mathrm{~m}$; à partir de $52 \mathrm{~m}$, la glace provient d'une zone située à environ $900 \mathrm{~km}$ de la côte, cette origine étant repoussée, pour les valeurs extrêmes, à $1000 \mathrm{~km}$.

L'analyse chimique confirme donc l'existence de deux glaces très différentes à la station G 1 . Les teneurs de la glace d'origine locale sont en accord avec celles mesurées dans les stations voisines. Par contre, la glace d'origine lointaine contient environ, en moyenne, 3 fois moins d'impuretés.

Ceci peut être dû soit à une variation de la teneur en impuretés des précipitations au cours des âges, puisque la glace formée à $1000 \mathrm{~km}$ pourrait s'être déposée il y a 50000 ans $^{1}$ (P. A. Shumskiy, communication personnelle), soit à une décroissance de la teneur en impuretés dans l'intérieur du continent.

Bien qu'il soit difficile de discuter la première de ces causes, il semble raisonnable de penser que le facteur géographique est le plus important, puisqu'il a déjà été montré que la concentration des ions dans l'Antarctique décroît lorsqu'on s'éloigne de la mer (Matveev, 1961).

\subsection{Origine des éléments}

En général, la détermination de l'origine des éléments contenus dans les précipitations est abordée par la comparaison des rapports de concentration avec ceux mesurés dans l'eau de mer, milieu dont la composition est pratiquement constante. Les valeurs moyennes trouvées dans notre cas sont données dans le Tableau 2;

1 Cette donnée a été calculée en admettant un axe de symétrie et un état d'équilibre pour l'An. tarctique. 

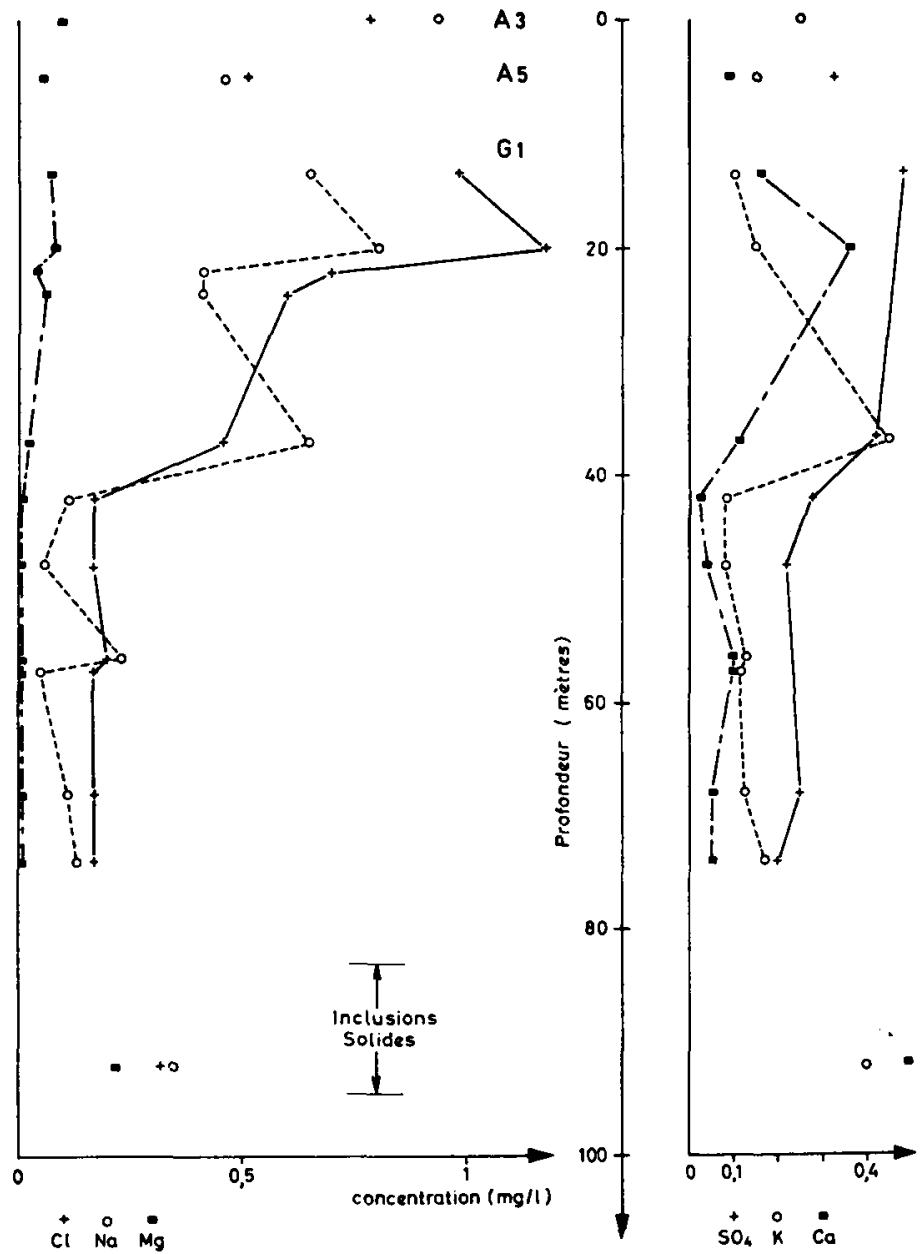

Fig. 2. Concentrations (mg/l) en fonction de la profondeur.

le nombre de mesures où existait une valeur définie pour chacun des éléments du rapport est indiqué entre parenthèses.

On note ainsi que les valeurs des rapports de concentration $\mathrm{Cl} / \mathrm{Ca}, \mathrm{Cl} / \mathrm{K}$ et $\mathrm{K} / \mathrm{Na}$ s'écartent des valeurs correspondantes pour l eau de mer d'un facteur compris entre 4 et 50. Par contre, ce facteur est toujours inférieur à 3 pour les rapports $\mathrm{Cl} / \mathrm{Na}, \mathrm{Cl} / \mathrm{Mg}$ et $\mathrm{Mg} / \mathrm{Na}$, un certain nombre de valeurs trouvées dans la glace étant très voisines de cells observées dans l'eau de mer. Cette différence est confirmés par le calcul des moyennes des rapports de concentration d'un même élément dans l'eau de mer et dans la glace (Tableau 4). Pour les stations A3, A5 et partie supérieure de GI, d'une part, partie inférieure de G 1 d'autre part, on voit ainsi que la dilution par rapport à l'eau de mer est du même ordre de grandeur pour les ions $\mathrm{Cl}$, Na et $\mathrm{Mg}$ alors qu'elle est nettement moins forte pour les ions $\mathbf{K}$ et $\mathrm{Ca}$. De plus, il faut noter que les normalités des principaux anions et cations que nous avons déterminés ne sont pas équivalentes, en opposition avec ce qui est observé dans le cas de l'eau de mer.

Si l'on rappelle que les chaînes de montagne de l'Antarctique les plus proches sont ì plus de $1000 \mathrm{~km}$ et l'Australie à près de $3000 \mathrm{~km}$ de l'emplacement où ont été effectués les prélèvements, que les ions déterminés sont les plus abondants de ceux existant dans l'eau de mer et que leur concentration décroît dans les pré-

Tellus XXI (1969), 1 
Tableau 3. Concentrations moyennes ( $m g / l)$

\begin{tabular}{|c|c|c|c|c|c|c|c|}
\hline & $\mathrm{Cl}$ & $\mathrm{Na}$ & $\mathbf{M g}$ & $\mathbf{K}$ & $\mathrm{Ca}$ & $\mathrm{SO}_{4}$ & $\mathrm{SiO}_{2}$ \\
\hline A 3 & 0,79 & 0,94 & 0,11 & 0,25 & - & - & - \\
\hline A 5 & 0,49 & 0,46 & 0,05 & 0,15 & 0,09 & 0,33 & 0,03 \\
\hline G $10-40 \mathrm{~m}$ & 0,79 & 0,59 & 0,06 & 0,23 & 0,21 & 0,45 & 0,04 \\
\hline $40-80 \mathrm{~m}$ & $\begin{array}{l}<0,19 \\
=0,14^{a}\end{array}$ & 0,11 & $(0,009-0,011)$ & $(0,10-0,12)$ & $(0,035-0,065)$ & 0,24 & 0,05 \\
\hline
\end{tabular}

a Si l'on prend $\mathrm{Cl} / \mathrm{Na}=1,3$ valeur mesurée à $55 \mathrm{~m}$.

cipitations lorsqu'on s'éloigne de la côte, il est raisonnable de penser à une origine océanique pour ces éléments contenus dans la neige. Pourtant, par rapport au chlore dont on peut supposer qu'il est entièrement d'origine marine et aux concentrations relatives dans l'eau de mer, il existe un excès de teneur pour les différents ions. Ceci pourrait être dû à un enrichissement des précipitations, non seulement en potassium (Wilson, 1959; Odie, 1960) mais aussi en différents ions en fonction de leur poids atomique (Komabayasi, 1962). On peut aussi penser à une perte préférentielle du chlore dans l'atmosphère (Junge, 1963) ce qui expliquerait les valeurs un peu faibles du rapport $\mathrm{Cl} / \mathrm{Na}$; dans cette dernière hypothèse, il faudrait alors envisager un apport d'origine différente pour les cations $\mathrm{Ca}$ et $\mathrm{K}$.

D'un autre côté, il est curieux de constater que les excès calculés pour les ions $\mathrm{Na}, \mathrm{K}$ et $\mathrm{Ca}$ sont du même ordre de grandeur. Cette abondance relative est conforme à la composition moyenne de la croûte terrestre, ce qui suggère une origine possible pour ces ions malgré l'éloig. nement des sources et les faibles valeurs trouvées pour la silice. Cette possibilité est à rapprocher des résultats trouvés dans les régions centrales du Groenland (Junge, 1963; Langway, 1967) où l'on a noté la présence de matière minérale. Ainsi, à la présence d'ions $(\mathrm{Cl}, \mathrm{Na}$ et $\mathbf{M g}$ ) d'origine marine s'ajouterait un apport d'ions ( $\mathrm{Na}, \mathrm{K}$ et $\mathrm{Ca}$ ) provenant directement de la croûte terrestre. Enfin, il reste la possibilité d'un apport d'origine extra-terrestre.

Par ailleurs, les fortes concentrations mesurées pour les sulfates, notamment dans la glace d'origine lointaine, où ces ions deviennent nettement prédominants, confirme l'existence d'un excès d'ions $\mathrm{SO}_{4}$ même dans les régions non polluées. La concentration mesurée pour le fond de G $1(0,25 \mathrm{mg} / \mathrm{l})$ est en effet comparable aux valeurs obtenues au centre du Groenland par Junge (1960) qui trouve $0,25 \mathrm{mg} / \mathrm{l}$ et Langway (1967) qui donne pour la moyenne de plusieurs stations $0,29 \mathrm{mg} / \mathrm{l}$. Dans l'Antarctique, les données de Matveev (1961) pour les régions centro. les sont sensiblement les mêmes.

\subsection{Variations fines}

L'examen des analyses effectuées sur des échantillons fins montre la très grande dispersion de la concentration des différents éléments et de leurs rapports. Cette dispersion reflète probablement la complexité des phénomènes mis en jeu : origine des différents ions, processus de circulation et de déposition.

A titre d'exemples, on donne les variations observées en $A_{3}$ (Fig. 3). Pour cette étude, on a

Tableau 4. Rapport des concentrations $\frac{m g / l \text { eau de mer }}{m g / l \text { glace }} \cdot 10^{3}$.

\begin{tabular}{lcllllr}
\hline & $\mathrm{Cl}$ & $\mathrm{Na}$ & $\mathrm{Mg}$ & $\mathrm{K}$ & $\mathrm{Ca}$ & $\mathrm{SO}_{\mathbf{4}}$ \\
\hline A 3 & $\mathbf{2 4}$ & 11,2 & 11,5 & $\mathbf{1 , 5}$ & - & - \\
A 5 & $\mathbf{3 8 , 7}$ & $\mathbf{2 3}$ & $\mathbf{2 5 , 4}$ & $\mathbf{2 , 5}$ & $\mathbf{4 , 4}$ & $\mathbf{8}$ \\
G 10-40 m & $\mathbf{2 4}$ & $\mathbf{1 8}$ & $\mathbf{2 2 , 3}$ & 1,6 & $\mathbf{1 , 9}$ & $\mathbf{5 , 8}$ \\
$40-80 \mathrm{~m}$ & $>100$ & 96 & 127 & $\mathbf{3 , 5}$ & $\mathbf{8}$ & 11 \\
\hline
\end{tabular}




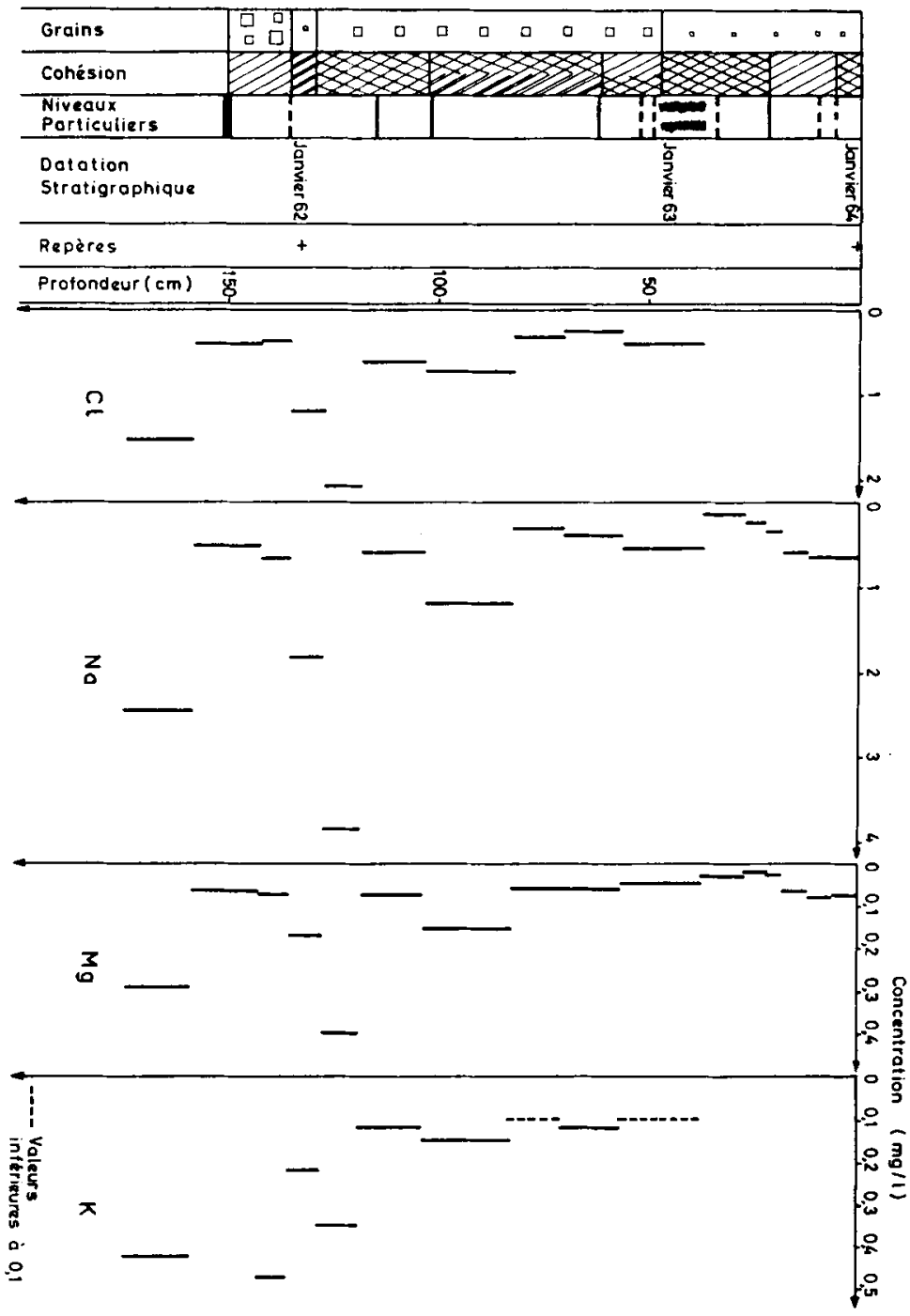

Fig. 3. Variation fine des concentrations $(\mathrm{mg} / \mathrm{l})$ à la station A 3.

choisi d'effectuer les mesures sur des échantillons de l'ordre de $3 \mathrm{~cm}$ d'épaisseur prélevés de façon continue, cette épaisseur étant très inférieure à l'accumulation qui est de l'ordre de $45 \mathrm{~cm}$ de névé par an et nous disposons de différentes observations qui ont été reportées Fig. 4; on constate que si les variations de concentrations des ions $\mathrm{Cl}, \mathrm{Na}$ et $\mathrm{Mg}$ sont comparables, celles des ions ions $\mathrm{K}$ et $\mathbf{C a}$ (données non publiées) sont différentes. De plus, sur une période de 2 années, ces variations n'ont pû Tellus XXI (1969). I être liées avec l'alternance saisonnière déterminée à partir de l'étude stratigraphique et des repères d'accumulation, comme aurait pû le laisser espérer la présence d'une mer gelée en hiver. Il est vrai qu'en Terre Adélie de nombreux facteurs peuvent intervenir tels qu'une redistribution des précipitations sous l'action du vent et des débacles de glace de mer en hiver. $\mathrm{Ce}$ problème nécessiterait pourtant de nouvelles études, en particulier dans les régions où le vent est moins violent, bien que d'autres 
travaux réalisés dans l'Antarctique (Brocas \& Delviche, 1963) et au Groenland (Langway, 1967) n'aient pas apporté de résultats positifs.

A la station $\mathrm{G} 1$, on a constaté que l'hétérogénéité des différentes couches est conservée et ceci même pour les couches profondes constituées par de la glace qui s'est écoulée depuis les parties centrales du continent.

\subsection{Autres mesures}

En plus des ions les plus importants dont les résultats ont été exposés ci-dessus, un certain nombre de déterminations ont porté sur les éléments suivants: $\mathrm{Ba}, \mathrm{B}, \mathrm{Co}, \mathrm{Cr}, \mathrm{Ni}, \mathrm{Ti}$ et $\mathrm{Sn}$. Dans l'eau de fusion, la concentration de ces éléments a toujours été trouvée inférieure à 0,01 p.p.m. Dans quelques cas, on a trouvé des teneurs en $\mathrm{Al}$ jusqu'à 0,04 p.p.m., en Fe jusqu'à 0,16 p.p.m., en Mn jusqu'è 0,025 p.p.m. et en Zn jusqu'à 0,05 p.p.m.; la concentration était un peu plus élevée dans la glace contenant des poussières morainiques.

En une station (A 5), on a dosé les matières organiques : la moyenne de six déterminations conduit à des équivalents en $\mathrm{ml}$ de $\mathrm{MnO}_{4} \mathrm{~K}$ (solution à N/1000) par gramme de glace de $0,10 \pm 0,05$ à froid et $0,14 \pm 0,05$ à chaud.

Différentes déterminations ont aussi été faites sur des échantillons provenant d'icebergs et de la glace bleue du fond du forage G1. Pour les premiers, on a trouvé de façon très nette que les couches superficielles étaient recouvertes par des embruns d'origine marine, les teneurs en Na atteignant jusqu'à l $\mathrm{g}$ par litre. Dans le second cas, les analyses ont été effectuées sur les mêmes prélèvements que ceux ayant été utilisés pour la mesure des teneurs en isotopes stables. L'accident trouvé pour les teneurs on deutérium ne peut être interprété (Merlivat et al., 1966) qu'en admettant que les $2 \mathrm{~m}$ de glace bleue rencontrés en $G l$ avant le socle rocheux ont été formés par le gel de l'eau de mer au contact de la glace froide. Cette interprétation est en accord avec les différentes observations : il n'existe pas de trace de cette glace en surface alors que toutes les couches supérieures y sont représentées, absence d'inclusions gazeuses, socle rocheux situé à environ $10 \mathrm{~m}$ au-dessous du niveau de la mer; les cotes du socle rocheux diminuent d'ailleurs jusqu'à la vallée sousglaciaire voisine du glacier de l'Astrolabe (profondeur de l'ordre de $1500 \mathrm{~m}$ ) occupée actuellement par la mer (Lorius, Rouillon \& Helly,

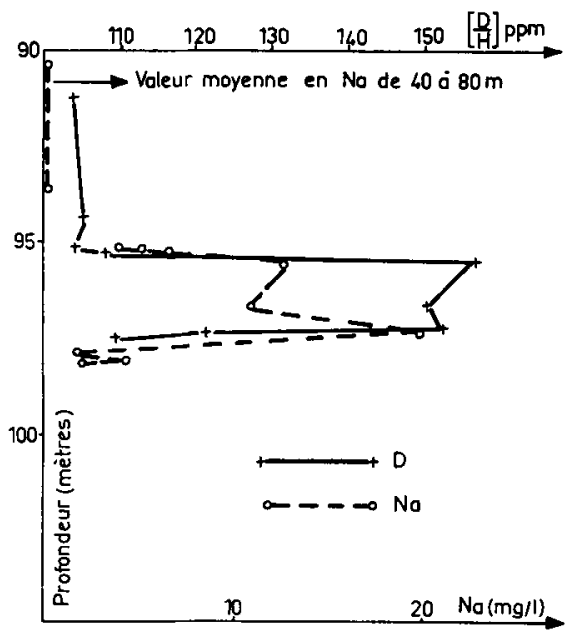

Fig. 4. Concentrations en $\mathrm{Na}(\mathrm{mg} / \mathrm{l})$ et en deutérium (p.p.m.) à la base du forage $\mathrm{Gl}$.

1967) ce qui permet de comprendre le passage de celle-ci jusqu'à l'emplacement du forage. De plus, la résistivité de l'eau de fusion (mesurée en présence d'air atmosphérique) est d'environ $7 \cdot 10^{\mathrm{s}} \Omega \cdot \mathrm{cm}$ alors qu'elle est généralement supérieure à $10^{5} \Omega \cdot \mathrm{cm}$ pour les couches supérieures.

Les résultats obtenus par l'analyse chimique confirment cette explication : les variations de teneur en deutérium sont parallèles à celles observées pour la teneur en $\mathrm{Na}$, les concentrations mesurées atteignant jusqu'à $20 \mathrm{mg} / \mathrm{l}$ (Fig. 4). Le fait que cette valeur soit relativement faible comparée aux teneurs habituellement trouvées pour la glace se formant à la surface de la mer peut s'expliquer par différentes raisons (Adams, French, Kingery, 1963) telles que la migration des sels durant des milliers d'années depuis lesquels cette glace serait formée (Merlivat et al., 1966) et le lavage continuel entrainant probablement une partie importante des impuretés an cours de la formation lente de cette glace au contact d'une paroi froide. Dans un cas semblable de gel d'eau de mer au contact de la glace, de tels ordres de grandeur ont d'ailleurs déjà été observés (Ragle, Blair et Person, 1964).

\section{Estimation des quantités se deposant sur l'Antarctique}

Si de nombreuses déterminations de concentration ont été faites sur les précipitations 
Tableau 5. Concentrations dans la glace en Antarctique (mg/l)

\begin{tabular}{|c|c|c|c|c|c|c|c|c|c|c|c|}
\hline Static & ons & $\begin{array}{l}\text { Alt. } \\
\text { (m) }\end{array}$ & $\begin{array}{c}\text { D. } \\
\text { côte } \\
(\mathbf{k m})\end{array}$ & $\mathbf{E}^{a}$ & $\mathrm{Cl}$ & $\mathbf{N a}$ & $\mathbf{K}$ & $\mathbf{C a}$ & $\mathbf{M g}$ & $\mathrm{SO}_{4}$ & Références \\
\hline $40^{\circ} \mathrm{E} 68^{\circ} \mathrm{S}$ & Base Showa & $\sim 0$ & $\sim 0$ & I & $\begin{array}{l}413 \\
8,3\end{array}$ & $\begin{array}{l}240 \\
5,3\end{array}$ & & $\begin{array}{l}8,9 \\
0,42\end{array}$ & $\begin{array}{l}20 \\
0,6\end{array}$ & $\begin{array}{l}46 \\
0,8\end{array}$ & $\begin{array}{l}\text { Sugarawa } \\
(1961)\end{array}$ \\
\hline $\begin{array}{r}95^{\circ} \mathrm{E} 67^{\circ} \mathrm{S} \\
79^{\circ} \mathrm{S}\end{array}$ & $\begin{array}{c}\text { Base Mirny } \\
\text { (à 50 km) } \\
\text { Base Vostok }\end{array}$ & $\begin{array}{r}800 \\
3420\end{array}$ & $\begin{array}{r}50 \\
1400\end{array}$ & 48 & $\begin{array}{l}2,61 \\
0,35\end{array}$ & \multicolumn{2}{|c|}{1,81} & $\begin{array}{l}0,68 \\
0,49\end{array}$ & $\begin{array}{l}0,17 \\
0,16\end{array}$ & $\begin{array}{l}0,46 \\
0,25\end{array}$ & $\begin{array}{c}\text { Matveev } \\
(1961)\end{array}$ \\
\hline $120^{\circ} \mathrm{W} 80^{\circ} \mathrm{S}$ & Base Byrd & 1510 & 700 & 2 & 0,14 & $\begin{array}{l}0,03 \\
0,16\end{array}$ & & & & & $\begin{array}{l}\text { Allis-Chal- } \\
\text { mers } 1963\end{array}$ \\
\hline $\begin{array}{l}24^{\circ} \mathrm{E} 70^{\circ} \mathrm{S} \\
70- \\
72^{\circ} \mathrm{S} \\
72^{\circ} \mathrm{S}\end{array}$ & $\begin{array}{l}\text { Cote vers base } \\
\text { Roi Baudoin } \\
\text { Continent } \\
\text { Montagnes }\end{array}$ & $\begin{array}{l}\quad 40 \\
\quad 0- \\
1200 \\
\sim 2000\end{array}$ & $\begin{array}{r}10 \\
0- \\
150 \\
\sim 200\end{array}$ & 100 & $\begin{array}{l}0,47 \\
0,39\end{array}$ & $\begin{array}{l}0,56 \\
1,07\end{array}$ & $\begin{array}{l}0,38 \\
0,17 \\
0,33\end{array}$ & & & & $\begin{array}{l}\text { Brocas \& } \\
\text { Delviche } \\
(\mathbf{1 9 6 3 )}\end{array}$ \\
\hline $\begin{array}{l}\text { Pôle Sud } \\
172^{\circ} \mathrm{E} 83^{\circ} \mathrm{S}\end{array}$ & $\begin{array}{l}\text { Glacier de } \\
\text { Beardmore }\end{array}$ & $\begin{array}{r}2800 \\
100\end{array}$ & $\begin{array}{r}1200 \\
800\end{array}$ & $\begin{array}{l}3 \\
1\end{array}$ & 0,027 & $\begin{array}{l}0,011 \\
0,13\end{array}$ & $\begin{array}{l}0,007 \\
0,05\end{array}$ & & & & $\begin{array}{l}\text { Wilson \& } \\
\text { House } \\
(\mathbf{1 9 6 5 )}\end{array}$ \\
\hline$\underbrace{163^{\circ} \mathrm{E} 77^{\circ} \mathrm{S}}$ & $\begin{array}{l}\text { Glacier Taylor } \\
\text { Glacier Wilson }\end{array}$ & & & $\begin{array}{l}1 \\
1\end{array}$ & $\begin{array}{r}561 \\
14\end{array}$ & $\begin{array}{l}\mathbf{0} \\
\mathbf{B}\end{array}$ & $\begin{array}{l}0 \\
4\end{array}$ & $\begin{array}{r}20 \\
7\end{array}$ & $\begin{array}{r}104 \\
<1\end{array}$ & $\begin{array}{r}38 \\
0\end{array}$ & $\begin{array}{l}\text { Angino, } \\
\text { Armitage } \\
\text { \& Tash } \\
(1965)\end{array}$ \\
\hline $\begin{array}{l}24^{\circ} \mathrm{E} 70^{\circ} \mathrm{S} \\
\text { Pole Sud }\end{array}$ & $\begin{array}{l}\text { vers Base Roi } \\
\text { Baudoin }\end{array}$ & $\begin{array}{r}40 \\
2800\end{array}$ & $\begin{array}{r}10 \\
1200\end{array}$ & 1 & $\begin{array}{r}0,63 \\
<0,001\end{array}$ & $\begin{array}{l}0,74 \\
0,003\end{array}$ & $\begin{array}{l}0,19 \\
0,008\end{array}$ & $\begin{array}{l}0,067 \\
0,008\end{array}$ & 0,006 & & $\begin{array}{l}\text { Brocas \& } \\
\text { Picciotto } \\
(1967)\end{array}$ \\
\hline $140^{\circ} \mathrm{E} 67^{\circ} \mathrm{S}$ & $\begin{array}{c}\text { vers Base } \\
\text { Dumont } \\
\text { d'Uryille }\end{array}$ & 230 & 5 & 73 & 0,69 & 0,66 & 0,21 & 0,15 & 0,07 & 0,39 & Présent \\
\hline $130^{\circ} \mathrm{E} 73^{\circ} \mathrm{S}$ & $\begin{array}{l}\text { d'apres resul- } \\
\text { tats G l }\end{array}$ & 3000 & 800 & 26 & 0,14 & 0,11 & 0,11 & 0,05 & 0,01 & 0,24 & travail \\
\hline
\end{tabular}

a $\mathbf{E}=$ échantillons.

dans différentes parties du globe, les observations concernant les régions polaires sont relativement peu nombreuses. Pour le Groenland, citons les travaux de Junge $(1960,1963)$ et de Langway (1962, 1965, 1967).

En ce qui concerne l'Antarctique, les données disponibles à notre connaissance sont résumées dans le Tableau 5. De cet ensemble, il semble difficile d'utiliser celles de la base Showa et des glaciers Taylor et Wilson (présence probable de contamination naturelle); de plus, les valeurs trouvées à la base Mirny (Matveev, 1963) et non citées dans le tableau, semblent très influencées par la proximité immédiate de la mer. A un degré bien moindre, ceci est peut-être aussi valable pour la première série de déterminations faite sur la côte, vers la base Roi Baudoin (Brocas \& Delviche, 1963) si on la compare avec les valeurs publiées ultérieurement (Brocas
\& Picciotto, 1967). Notons enfin les apports d'origine locale signalés par les auteurs dans les régions montagneuses vers $20^{\circ} \mathrm{E}$ et $72^{\circ} \mathrm{S}$ (Brocas \& Delviche, 1963) notamment pour le sodium. Nous nous bornerons donc à discuter les résultats en italiques dans le Tableau 5 qui indiquent une décroissance très nette des concentrations en fonction de la distance à la côte; compte tenu du fait que les ions $\mathrm{Cl}$ et $\mathrm{Na}$ sont quantitativement les plus importants, il nous a été ainsi possible d'avoir une idée de la décroissance de la somme des concentrations, en ce qui concerne les ions $\mathrm{Cl}, \mathrm{Na}, \mathrm{K}, \mathrm{Ca}, \mathrm{Mg}$ (Fig. 5). Dans cette figure, il n'a pas été tenu compte des résultats publiés par Matveev (1963) car les concentrations obtenues lo long du méridien $95^{\circ} \mathrm{E}$ entre les stations Mirny et Vostok sont très supérieures. Sans parler de causes d'erreurg accidentelles toujours possibles, 


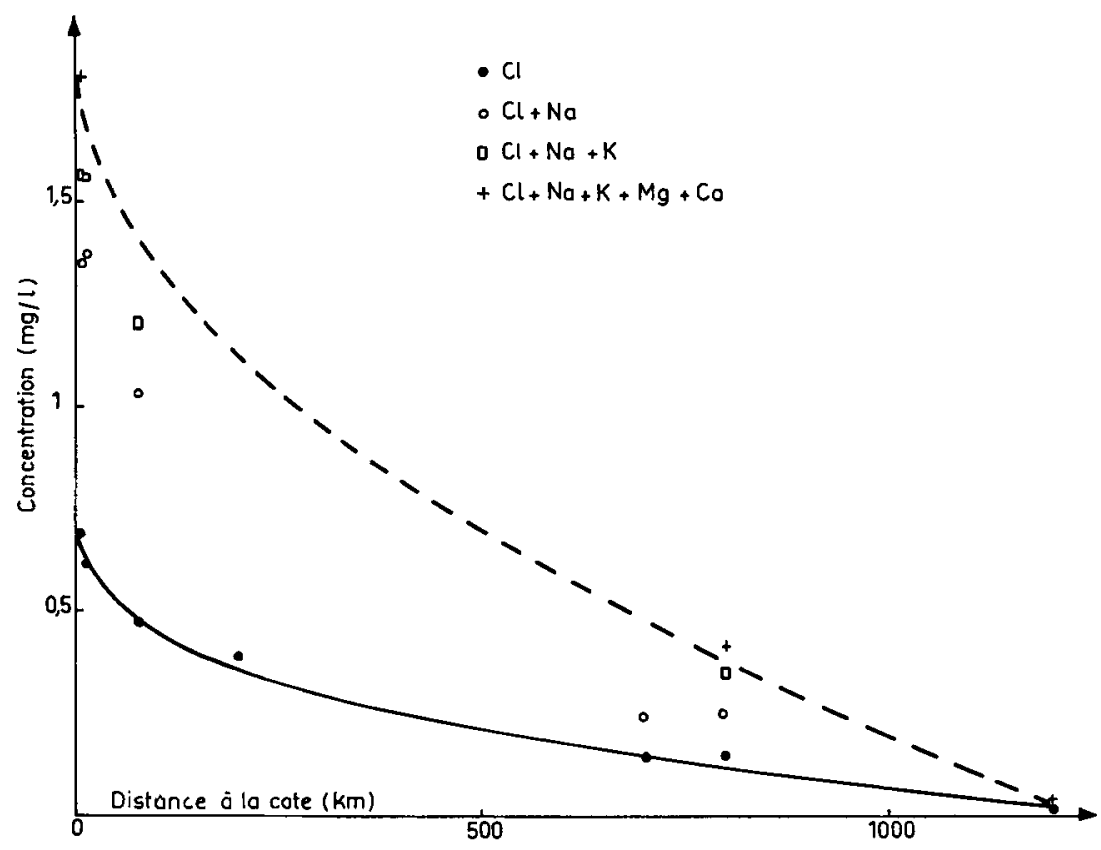

Fig. 5. Concentration de la glace de l'Antarctique $(\mathrm{mg} / \mathrm{l})$ en fonction de la distance à la côte $(\mathrm{km})$.

on peut envisager que ces fortes valeurs soient dues à un aspect particulier de la circulation atmosphérique dans ce secteur. En effet, si l'on consulte une carte des accumulations annuelles de neige dans l'Antarctique (Bentley, Cameron, Bull, Kojima \& Gow, 1964) on constate la présence d'une anomalie particulièrement marquée dans les régions côtières situées vers la base Mirny. Cette anomalio est caractérisée par de très fortes valeurs qui peuvent atteindre jusqu'à $1 \mathrm{~m}$ d'eau par an et qui sont vraisemblablement liées à un régime particulier de précipitations qui seraient alors riches en impuretés. Pour en revenir aux remarques faites au sujet des sulfates, il est intéressant de noter ici que la concentration de cet ion est, dans ce cas, sensiblement peu différente de celles que nous avons mesurées, au contraire des autres éléments.

A partir de la Fig. 5 et de la carte de l'accumulation moyenne annuelle de neige sur l'Antarctique (Bentley et al., 1964) on peut alors attribuer à chaque tranche d'accumulation définie sur ce document une valeur moyenne approximative de concentration pour la somme de ces ions, puisque l'accumulation décroît elle aussi de façon relativement régulière en fonction de la distance à la côte. Pour chacune de ces zones d'accumulation, on multiplie alors la concentration par les volumes d'eau tombant chaque année, la quantité de sulfates étant considérée indépendamment puisque les ions $\mathrm{SO}_{4}$ varient en effet de façon beaucoup moins nette. Nous obtenons ainsi, pour les ions $\mathrm{Cl}$, $1,1 \cdot 10^{6}$ tonnes et pour la somme des ions $\mathrm{Cl}$,

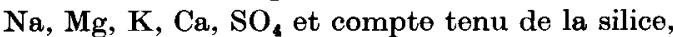
environ $4 \cdot 10^{6}$ tonnes, quantité tombant chaque année sur l'Antarctique, soit une surface de l'ordre de $13,5 \cdot 10^{\circ} \mathrm{km}^{2}$, et pour un volume de précipitations de $2300 \mathrm{~km}^{3}{ }^{1}$

Comme les considérations précédentes pouvaient le laisser prévoir, ces estimations sont inférieures à celles données par Matveev (1962) à partir des déterminations effectuées sur le profil Mirny-Vostok, bien que les ordres de grandeur soient tout de même comparables. Celles-ci sont en effet de $6 \cdot 10^{6}$ tonnes/an pour le chlore et de $20 \cdot 10^{6}$ tonnes par an pour l'ensemble des ions.

Pour ces évaluations, nous avons tenu compte des éléments principaux habituellement déterminés dans les précipitations (Junge, 1963) à

1 Il n'est pas tenu compte de la péninsule antarctique dans cette estimation. 
l'exception des ions $\mathrm{CO}_{3} \mathrm{H}, \mathrm{NO}_{2}$ et $\mathrm{NO}_{3}, \mathrm{NH}_{4}$. Les concentrations en ions $\mathrm{CO}_{3} \mathrm{H}$ devraient être négligeables si les carbonates se trouvaient dans les mêmes proportions que dans l'eau de mer; pourtant, les déterminations faites par Matveev (1961) montrent des teneurs importantes peut-être influencées par le fait qu'une partie des carbonates provient du $\mathrm{CO}_{2}$ de l'air dissous dans l'eau. Par contre, les mesures de Wilson \& House (1965) montrent que les teneurs en ions $\mathrm{NH}_{4}$ sont pratiquement négligeables et que la concentration des ions $\mathrm{NO}_{2}+\mathrm{NO}_{3}$ qui seraient produits dans la haute atmosphère est de $0,005 \mathrm{mg} / \mathrm{l}$, valeur très inférieure à celles obtenues pour les autres éléments. Cette quantité de matière ne s'accumule pourtant pas chaque année sur l'Antarctique puisque les substances contenues dans la glace retournent ì l'océan sous différentes formes : eau de fusion, icebergs ... Même si le bilan de masse de l'Antarctique est approximativement en équilibre, il est à noter que ce cycle s'effectue de manière sélective suivant les éléments : les régions périphériques pour lesquelles la teneur en ions $\mathrm{Cl}$ et $\mathrm{Na}$ est prédominante sont, en effet, plus affectées par les différentes formes d'ablation que les régions centrales où prédominent les sulfates.

Si l'on admet qu'il n'y pas eu de variations importantes des éléments déposés au cours des âges (ce que semblent confirmer les valeurs que nous avons trouvées pour la vieille glace), on peut donner une estimation approximative de la quantité d'impuretés actuellement contenues dans l'Antarctique. Le calcul effectué à partir des valeurs que nous avons trouvées pour la partie inférieure du forage $G 1$, constituée par de la glace provenant des régions centrale, donne les résultats suivants en tonnes $\times 10^{\circ}$ :

$\begin{array}{lcccccc}\mathrm{Cl} & \mathrm{Na} & \mathrm{K} & \mathrm{Ca} & \mathrm{Mg} & \mathrm{SO}_{4} & \mathrm{SiO}_{2} \\ 3,7 & 2,9 & 2,9 & 1,3 & 0,3 & 6,4 & 1,3\end{array}$

soit un total de $18,8 \cdot 10^{9}$ tonnes pour un volume d'eau de 26,5 $10^{8} \mathrm{~km}^{3}$ (Bauer, 1961).

Compte tenu des volumes que représentent l'Antarctique et le Groenland, cette valeur est cohérente avec celle donnée dans ce dernier cas par Langway (1967) : 2089.10 $10^{6}$ tonnes pour un volume d'eau de $2,35 \cdot 10^{8} \mathrm{~km}^{3}$.

\section{Résumé}

On a étudié la concentration de différents ions dans la glace (jusqu'à $100 \mathrm{~m}$ de profondeur) et le névé de la région côtière de Terre Adélie. On constate que la teneur des couches supérieures de la glace est sensiblement la même que celle des couches de névé accumulées localement; les principaux ions, dont la concentration moyenne ost toujours inférieure à $\mathrm{l} \mathrm{mg} / \mathrm{l}$, sont dans l'ordre $\mathrm{Cl}, \mathrm{Na}$ et $\mathrm{SO}_{4}$, puis $\mathrm{K}$ et Ca. La glace des couches inférieures provenant, après écoulement, des régions intérieures de l'Antarctique contient environ 3 fois moins d'impuretés, la teneur en ions $\mathrm{SO}_{4}$ devenant alors prédominante $(0,24 \mathrm{mg} / \mathrm{l})$. On discute l'origine possible des différents ions, dont les variations importantes de concentration en une même station ne semblent pas liées à un phénomène saisonnier.

A partir des données publiées, on estime que se déposent chaque année sur l'Antarctique environ $4 \cdot 10^{6}$ tonnes d'impuretés solubles. Ces impuretés participent à un cycle mais la glace de ce continent pourrait en contenir $19 \cdot 10^{\circ}$ tonnes.

\section{REFERENCES}

Allis-Chalmers Co. 1964, cité par Mellor, M. Properties of snow. $C R R E L$, III.A 1, $105 \mathrm{p}$.

Adams, C. M. French, D. N. \& Kingery, W. D. 1963. Field solidification and desalinisation of sea ice. Ice and Snow, M.I.T. Press, Cambridge, p. 277288.

Angino, E. E., Armitage, K. B. \& Tash, J. C. 1965. Ionic content of Antarctic ice samples. Polar Record 12 (79), p. 407-409.

Bauer, A. 1961. Nouvelle estimation du volume de glace de l'Inlandsis Antaretique. AIHS 55, 19-23.

Bentley, C. R., Cameron, R. L., Bull, C., Kojima, $\mathbf{K}$. \& Gow, A. J. 1964. Physical characteristics of the Antarctic ice-sheet. Ant. Map Folio series, Folio 2, Amer. Geog. Soc.
Brocas, J. \& Delviche, R. 1963. $\mathrm{Cl}, \mathrm{K}$ and $\mathrm{Na}$ concentrations in Antarctic snow and ice. Jour. Geoph. Res. 68 (13), 3999-4000.

Brocas, J. \& Picciotto, E. 1967. Nickel content of Antarctic snow, implications on the influx rate of extraterrestrial dust. Jour. Geoph. Res., 72 (8), 2229-2236.

Charlot, G. 1966. Les méthodes de la chimie analytique. Analyse quantitative minérale. Masson, Paris.

Junge, C. E. 1960. Sulfur in the atmosphere. Jour. Geoph. Res. 65 (1), 227-237.

Junge, C. E. 1963. Air Chemistry and Radioactivity, Int. Geophysics series, vol. 4. Acad. Press, New York, 382 p. 
Komabayasi, M. 1962. Enrichment of inorganic ions with increasing atomic weight in aerosol, rainwater and snow in comparison with sea water. Jour. Met. Soc. Japan 40 (1), 25-38.

Langway, C. C. 1962. Some physical and chemical investigations of a 411 meters deep Greenland ice core and their relationship to accumulation. AIHS 58, 101-118.

Langway C. C. 1967. Chemical composition studies in "Stratigraphic analysis of a deep ice core from Greenland". CRREL Research Report 77, 130 p.

Langway, C. C., Oeschger, H., Alder, B. \& Renaud, A. 1965. Sampling polar ice for radiocarbon dating. Nature 206 (4983), 500-501.

Lorius, C., Rouillon, G. \& Helly, F. 1967. Sur une móthode gravimétrique simplifiée de détermination de l'épaisseur de la glace en bordure de l'Antarctique. C.R. Acad. Sc., série B, 264, p. 266-269.

Matveev, A. A. 1961. Chemical composition of the snow along the profile Mirny-Vostok (en russe) Gidrokhimicheskiye Materialy 34, p. 3-11.

Matveev, A. A. 1962. Chemical balance of atmospheric precipitation salts in Antarctica (en russe). Antarktika, Dokl. Komis. 1961, p. 18-26.

Matveev, A. A. 1963. Chemical composition of atmospheric precipitation according to observa. tions at MIRNYY (en russe). Results of researches according to the IGY program, sect. II, 5, p. 100107.

Merlivat, L., Lorius, C. \& Nief, G. 1966. Etudes isotopiques d'un glacier en Antarctique. C.R. Acad. Sc., série B, 263, p. 414-416.

Merlivat, L., Lorius, C., Majzoub, M., Nief, G. \& Roth, E. 1966. Etudes isotopiques en profondeur d'un glacier en Antarctique, colloque sur l'emploi des radioisotopes en hydrologie. A.I.E.A., Vienne, p. 671-681.

Oddie, B. C. V. 1960. The variation in composition of sea salt nuclei with mode of formation. Quart. J. Roy Meteo. Soc. 86, 549-551.

Ragle, R. H., Blair, R. G. \& Person, L. E. 1964. Ice core studies of Ward Hunt Ice Shelf 1960. J. of Glaciology 5 (37), 39-59.

Sugawara, K. 1961. Chemistry of ice, snow and other water substances in Antarctica. AIHS 55, 49-55.

Wilson, A. T. 1959. Surface of the ocean as a source of air-borne nitrogenous material and other plant nutrients. Nature 184, 99-101.

Wilson, A. T. \& House, D. A. 1965. Chemical composition of South Polar Snow. Jour. Geoph. Res. 70 (22), 5515-5518.

\section{РАСТВОРИМЫЕ ПРИМЕСИ, СОДЕРЖАЩИЕСЯ В АНТАРКТИЧЕСКОМ ЛЬДУ}

Изучалась ғонцентрация различных ионов во льду (на глубинах до 100 м) и в фирне на побережьи Земли Адели. Доказано, что концентрация әтих ионов в верхних слоях льда суцественно та же, что и в слоях фирна, аккумулированных адесь же. Основными ионами, средняя концентрация которых всегда меньше Iмг/л, являютея в порядке распространенности $\mathrm{Cl}, \mathrm{Na}$ и $\mathrm{SO}_{4}$, затем $\mathrm{K}$ и Са. Лед более глубоких слоев, притекший из внутренних областей Антарктики, содержит приблизительно в три раза меньше примесей, причем основной примесью становятся ионы $\mathrm{SO}_{4}$ $(0,24$ мг/л). Обсуждается возможное происхождение этих ионов, заметные вариации концентрации которых не кажутся связанными с сезонными явлениями.

Из опубликованных данных оценивается, что каждый год в Антарктике осаждается около $4,10^{6}$ тонн растворимых примесей. Эти примеси участвуют в ледовом цикле, поэтому весь лед әтого континента может содержать $19,10^{9}$ тонн примесей. 